\title{
Hospital-Based Prevalence of Iron Deficiency Anemia among Pre-School Children in Dubai
}

Wafaa Faysal ${ }^{1}$, Abdul Rehman Z. Zaidi ${ }^{2,}{ }^{3}$, Sameer Al-Abdi ${ }^{4}$, Saad Alhumaid ${ }^{5}$, Maied Z. AlShehery ${ }^{6}$, Abbas Al Mutair 2, 7

1. Pediatrics, Dr. Sulaiman Al Habib Medical Group, Dubai, ARE 2. Internal Medicine, Dr. Sulaiman Al Habib Medical Group, Riyadh, SAU 3. Internal Medicine, Alfaisal University, Riyadh, SAU 4. Pediatrics, King Abdulaziz Hospital, Ministry of the National Guard-Health Affairs, Al-Ahsa, SAU 5. Infectious Disease, Ministry of Health, Riyadh, SAU 6. Palliative Care, King Fahad Medical City, Riyadh, SAU 7. Internal Medicine, University of Wollongong, Wollongong, AUS

Corresponding author: Abbas Al Mutair, abbas4080@hotmail.com

\begin{abstract}
Introduction: Iron deficiency anemia (IDA) is an internationally recognized leading cause of disability and contributes to childhood morbidity and mortality. The prevalence of IDA is higher in developing countries, especially in Arab countries, compared to the west.
\end{abstract}

Methods: To assess the prevalence of IDA, we analyzed the data of children aged between one to five years seen at Dr. Sulaiman Al-Habib Medical Group's tertiary care hospital in Dubai, United Arab Emirates (UAE) from 2016 to 2018.

Results: We found a high occurrence of IDA in male children and non-Emirati children.

Conclusion: Appropriate screening and iron supplementation are required to see a decline in the rate of IDA. Further nationwide studies are required to identify the highly prevalent and high-risk areas of IDA in the UAE.

Categories: Pediatrics, Epidemiology/Public Health, Hematology

Keywords: iron deficiency anemia, anaemia, iron deficiency, united arab emirates, dubai, prevalence, children, pediatrics, anemia, ida

\section{Introduction}

Anemia can be defined as a decreased count of hemoglobin, red blood cells, and with possible alteration of red blood cell morphology [1]. There are many types of anemias and they are driven by multiple factors. The most common type of anemia is iron deficiency anemia (IDA) which affects more than $25 \%$ of the world's population [2]. IDA is the third leading cause of disability worldwide and contributes to childhood morbidity and mortality. According to a report by the World Health Organization, the global burden of IDA in 2000 was

Review began 09/28/2020 Review ended 10/07/2020 Published 10/11/2020

\section{(c) Copyright 2020}

Faysal et al. This is an open access article distributed under the terms of the Creative Commons Attribution License CC-BY 4.0., which permits unrestricted use, distribution, and reproduction in any medium, provided the original author and source are credited.
1946.4 years of life lost, 9430.1 years lost due to disability, and 11376.5 disability-adjusted life years [3].

Iron is important for the development of the central nervous system, especially during the first two years of life, hence a deficiency can cause cognitive decline [4]. IDA is a diversely distributed disease that mainly affects children, female teenagers, pregnant and lactating women [5]. Rapidly growing children (from 0-15 years of age) may consume iron stores that accrue during gestation, which can physiologically lead to an absolute deficiency in ferritin, making them one of the highest risk prone population of contracting IDA [6]. IDA can also be associated with low birth weight and a higher risk of maternal and perinatal mortality [7]. Symptoms occur due to reduced oxygen delivery to body tissues and may include dyspnea, headache, lethargy, paleness, difficulty in concentration, and can affect motor and mental development [8]. Severe IDA can also increase the risk of premature delivery, low birth weight, mortality during labor, higher rates of infections, and cardiac failure $[9,10]$.

The prevalence of iron deficiency seems to be higher in resource-deprived and developing countries, especially in infants in Arab countries ( 72\%), compared to developed nations [11-13]. The incidence rate of IDA in infants in a suburb of the United Arab Emirates (UAE) was previously reported to be 23.2\% [14]. As data from our region is scarce, we investigated the prevalence and predictors of IDA in children aged one to five years.

\section{Materials And Methods}

We retrospectively reviewed records of 1595 preschooler children (1-5 years old) who were seen at Dr. Sulaiman Al-Habib Medical Group's private tertiary care hospital in Dubai, UAE from 2016 to 2018 to assess the prevalence of IDA. 
We recorded the nationality of each study children and later we dichotomized the nationality as Emirati and Non-Emirati for analysis. We extracted white blood cell (WBC) count, hemoglobin level, ferritin level, iron supplementation, age, and gender of each study children. We defined IDA according to the criteria of the Centers for Disease Control and Prevention; a hemoglobin concentration $<11 \mathrm{~g} / \mathrm{dl}$ and serum ferritin $<10$ $\mu \mathrm{g} / \mathrm{L}[15,16]$. As ferritin may increase in an infectious or inflammatory state, we excluded any children that had WBC > $10 \times 109 / \mathrm{L}$ as a surrogate for any possible infections. We also recorded information on which of the children were already on iron supplements. The Institutional Review Board approved the study without a need to obtain written consent from the parents (IRB Log No. RC19.04.35).

We divided the study sample into two groups. In group 1, we wanted to test if a hemoglobin level $<11$ with lower than normal mean corpuscular volume (MCV) and/or high red cell distribution width (RDW) can identify IDA alone. Similarly, if ferritin of $<20$ can alone identify IDA correctly with a condition that WBCs were not $>10,000$. In group 2, hemoglobin $<11$ and ferritin $<20$ together were used to predict IDA more accurately.

Data were analyzed using IBM Statistical Package for the Social Sciences (SPSS) version 24 (SPSS Inc, Chicago, IL). A value of $\mathrm{p}<0.05$ was considered statistically significant. Our response variable is IDA that is classified into whether iron deficiency is present or not. Since our response was binary, we performed binary logistic regression. We applied binary logistic regression taking the predictors as gender, nationality, supplement, and age. Moreover, gender and nationality were our categorical variables. After executing binary logistic regression, we obtained the odds ratio and p-value of each predictor/factor. The model estimates are presented with the adjusted odds ratios and $95 \%$ confidence interval (CI).

\section{Results}

As seen in Table 1, among 1595 children, 800 ( 50\%) belonged to group 1, among which 444 (55.5\%) were male and 356 (44.5\%) were female. 224 children belonged to group 2 which were $14 \%$ of the sample having both hemoglobin and ferritin less than 11 and 20, respectively. In this category, 15\% were male and 12\% were female. $22 \%$ of our total sample were Emirati children, $53 \%$ of them were classified into group 1 and $12 \%$ were categorized into group 2 . A total of $39 \%$ of the children were taking IDA supplements. $46 \%$ of the total children were less than two years old. $48 \%$ of the total sample belonged to group 1 and $19 \%$ fell under group 2. To overcome any bias in our sample and to improve the accuracy of the study, we used WBC as an exclusion variable, and those that were having a WBC count of $>10,000$ were excluded. We only included 1,136 cases ( $71 \%$ of the total sample) in the study, who had a confirmed diagnosis of IDA in a tertiary care hospital setting (Figure 1). The age ranged from one to five years. Overall, more male children were affected by IDA than females.

\begin{tabular}{|c|c|c|c|}
\hline \multicolumn{2}{|l|}{ Factors } & Group 1: $\mathrm{Hb}<11$ or Ferritin $<20$ & Group 2: $\mathrm{Hb}<11$ \& Ferritin <20 \\
\hline \multirow{2}{*}{ Gender } & Male (878) & $444(51 \%)$ & $135(15 \%)$ \\
\hline & Female (717) & $356(50 \%)$ & $89(12 \%)$ \\
\hline \multirow{2}{*}{ Nationality } & Emirati (355) & $189(53 \%)$ & $64(18 \%)$ \\
\hline & Non-Emirati (1240) & $610(49 \%)$ & $160(13 \%)$ \\
\hline \multirow{2}{*}{ WBC } & $<10,000(1136)$ & $558(49 \%)$ & $196(12 \%)$ \\
\hline & $>10,000(459)$ & 242 (15\%) & $28(2 \%)$ \\
\hline \multirow{2}{*}{ IDA Supplement } & Yes (619) & $619(100 \%)$ & $43(7 \%)$ \\
\hline & No (976) & $52(5 \%)$ & $52(5 \%)$ \\
\hline \multirow{4}{*}{ Age } & $1-<2(735)$ & $355(48 \%)$ & $143(19 \%)$ \\
\hline & $2-3(317)$ & $147(46 \%)$ & $29(9 \%)$ \\
\hline & $3-4(320)$ & $162(51 \%)$ & $35(11 \%)$ \\
\hline & $4-5(223)$ & $136(61 \%)$ & $17(8 \%)$ \\
\hline
\end{tabular}

TABLE 1: Count and percentages of factors of $\mathrm{Hb}<11$ or ferritin $<20$ and $\mathrm{Hb}<11$ and ferritin $<20$

Hb: hemoglobin; WBC: white blood cell; IDA: iron deficiency anemia. 


\section{Cureus}

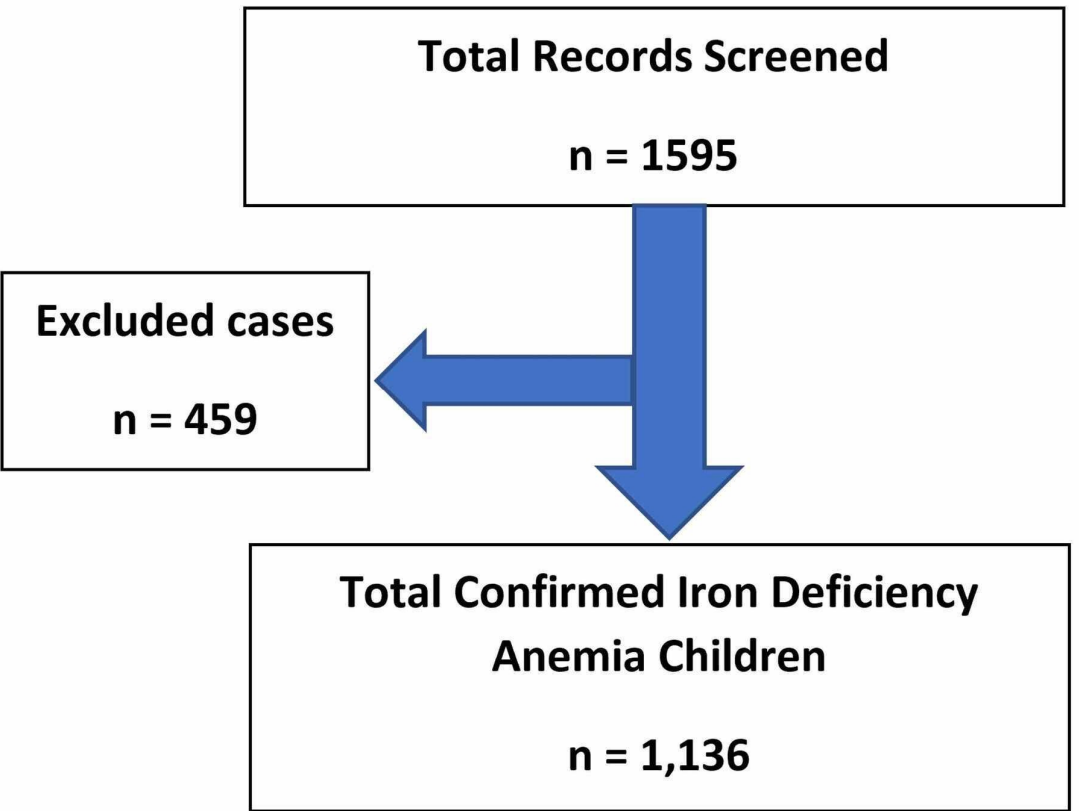

FIGURE 1: Exclusion of cases with white blood cell (WBC) $>10,000 x$ 10^9/L

The results of the binary logistic regression analysis can be seen in Table 2 . We can see that $57 \%$ of males had IDA and 58\% were in the non-IDA group. If we compare Emirati children with Non-Emirati children, $24 \%$ had IDA. There was no difference in the confidence intervals of children taking a supplement or not. The gender odds ratio (OR) was 0.977 with a p-value of 0.869 which means males were $3 \%$ more likely to have IDA, but gender is insignificant. Nationality OR was 1.539 with a significant p-value, which means Emirati children were 54\% less likely to have IDA in contrast to non-Emirati children. Supplement OR was 0.872 which means those that were taking supplements had a $12.8 \%$ more chance of having IDA, but supplements are insignificant at a $5 \%$ level of significance. Age OR is 1.001 showing that age did not affect the prevalence of IDA.

\begin{tabular}{|c|c|c|c|c|c|c|c|c|c|}
\hline \multirow{2}{*}{ Factors } & & \multirow{2}{*}{ IDA Count } & \multicolumn{2}{|l|}{ IDA 95\% CI } & \multirow{2}{*}{ Non-IDA Count } & \multicolumn{2}{|c|}{ Non-IDA 95\% Cl } & \multirow{2}{*}{ OR } & \multirow{2}{*}{ P-value } \\
\hline & & & Hemoglobin & Ferritin & & Hemoglobin & Ferritin & & \\
\hline \multirow{2}{*}{ Gender } & Male & 350 (57\%) & $11.30-11.93$ & $17.91-28.84$ & 305 (58\%) & $12.11-12.29$ & $39.87-44.97$ & \multirow{2}{*}{0.977} & \multirow{2}{*}{0.869} \\
\hline & Female & $260(43 \%)$ & $11.15-11.70$ & $20.07-27.68$ & 221 (42\%) & 12.08-12.31 & $46.75-61.40$ & & \\
\hline \multirow{2}{*}{ Nationality } & Emirati & $146(24 \%)$ & 10.97-11.50 & $19.05-36.29$ & 109 (21\%) & $11.90-12.23$ & $40.12-59.01$ & \multirow{2}{*}{1.539} & \multirow{2}{*}{$<0.01$} \\
\hline & Non-Emirati & $454(76 \%)$ & $11.35-11.91$ & $18.69-23.46$ & 417 (79\%) & $12.16-12.31$ & $43.07-50.14$ & & \\
\hline \multirow{2}{*}{ Supplement } & Yes & $330(54 \%)$ & $11.27-11.71$ & $17.66-24.56$ & $271(52 \%)$ & 12.11-12.32 & 41.81-50.92 & \multirow{2}{*}{0.872} & \multirow{2}{*}{0.321} \\
\hline & No & $280(46 \%)$ & $11.37-11.75$ & $16.59-23.10$ & $255(48 \%)$ & $12.09-12.27$ & 43.03-53.19 & & \\
\hline Age & & $610(100 \%)$ & $11.32-11.75$ & $19.91-25.50$ & 526 (100\%) & $12.16-12.44$ & $43.06-48.93$ & 1.001 & 0.795 \\
\hline
\end{tabular}

TABLE 2: Odds ratio (OR) and confidence interval (CI) of iron deficiency anemia (IDA) and noniron deficiency anemia

\section{Discussion}

Globally, iron deficiency is the most common nutritional disease and the root cause of anemia in children and pregnant women [17]. IDA commonly accompanies chronic illnesses such as chronic kidney disease, malignancy, rheumatoid arthritis, obesity, and inflammatory bowel disease [18-20]. The study of the prevalence of anemia, in general, is difficult as it requires an understanding of the epidemiology of the 
underlying causes of anemia. Therefore it is a good idea to study specific types of anemia, such as IDA, and also different severities of anemia [21]. Most studies from around the world focus on IDA due to its clinical significance and high prevalence. High-risk populations may have a total anemia occurrence of 50\%-80\%, and this may be due to a substandard socioeconomic status, lower body weight, and is found to be higher in new mothers [22].

In our study, we used high WBC as a surrogate marker for possible infection or acute phase reactive state that might lead to ferritin leak from the liver, falsely elevating ferritin levels, and missing the true iron deficiency state [23]. We utilized hemoglobin and ferritin levels, as ferritin is found to be a more accurate marker of IDA than traditional red cell indices, which in IDA, usually indicate that the red blood cells are microcytic and hypochromic. We found 1,024 children, with ages ranging from one-five years, to have IDA, which demonstrates a high prevalence of IDA in children in the UAE. Our findings are similar to studies done in Saudi Arabia [24,25], but show a lower incidence of IDA than a study done in Jordan [12]. The mean age of children with IDA was a year higher in our study than the mean age of 15.4 months in Saudi Arabia [24]. As previously reported in the Eastern Mediterranean region, we also observed a slightly higher incidence of IDA in male children [24,26].

We found a higher rate of IDA in non-Emirati children, and this may be because of the population of the Emiratis is lower than the expatriates living in the UAE. In 2011, it was reported that less than 1 million out of the 8.3 million residents of the UAE were Emirati [27]. We also observed the children on iron supplements had more odds of having IDA, and this might be because our study was of a retrospective design, and the children might have been already identified as IDA and those children may have been taking supplements after diagnosis of IDA.

Routine screening for anemia at 12 months of age is vital as recommended by the American Academy of Pediatrics and the World Health Organization [28]. Serum ferritin levels also need to be measured during screening to identify IDA, which if supplemented with dietary iron early, can potentially prevent cognitive defects. However, we must be cautious that the serum ferritin level is not the sole basis of diagnosis and that the child does not have an infection or any inflammatory disorder. Patients and physicians should be wary of children with poor growth, feeding problems, and inadequate dietary iron intake, as these children will need to be screened for IDA as well [29]. Routine screening for IDA is especially crucial for children living in areas with a high prevalence of IDA. This is also why national epidemiological studies to identify these regions are imperative.

To overcome iron deficiency in children, it is also essential to optimize their nutrition with targeted daily iron supplementation. Full-term breastfed infants can be started on elemental iron $1 \mathrm{mg} / \mathrm{kg}$ daily at four months of age and this can be sustained until the infant is on adequate iron-rich foods, such as meat, fortified food, and green vegetables. Such complementary iron-rich foods can be taken by six months of age [30]. Appropriate iron supplementation in children is vital in high-risk and highly prevalent areas of IDA. For children from the age of one to three years, the recommended dietary allowance for iron is $7 \mathrm{mg} / \mathrm{day}$ [30].

Our study had several limitations. Firstly, the cross-sectional nature of the study means that the chronological relationship between IDA and the associated factors cannot be established. We also could not include red cell indices of the children, which could have given us a better insight into the red blood cell morphology of the children. We also could not obtain the maternal red cell indices, nor their hemoglobin and ferritin levels to assess if the mothers also had IDA. Furthermore, we were unable to measure serum vitamin B12 and folate levels in these children and so could not quantify other forms of anemia, signifying some misclassification bias.

\section{Conclusions}

IDA is a serious concern and affects children worldwide, including the UAE. More children that were less than two years of age, non-Emirati, and males were affected by IDA in our findings. Rigorous screening and iron supplementation programs are necessary to see a significant decrease in this ailment. Further collaborative national studies are required to identify the high-risk and high-prevalence areas of IDA in different parts of the world.

\section{Additional Information \\ Disclosures}

Human subjects: All authors have confirmed that this study did not involve human participants or tissue. Animal subjects: All authors have confirmed that this study did not involve animal subjects or tissue. Conflicts of interest: In compliance with the ICMJE uniform disclosure form, all authors declare the following: Payment/services info: All authors have declared that no financial support was received from any organization for the submitted work. Financial relationships: All authors have declared that they have no financial relationships at present or within the previous three years with any organizations that might have an interest in the submitted work. Other relationships: All authors have declared that there are no other relationships or activities that could appear to have influenced the submitted work. 


\section{Acknowledgements}

The authors would like to thank the Research Center and IRB of Dr. Sulaiman Al Habib Medical Group for their tremendous support.

\section{References}

1. Vieth JT, Lane DR: Anemia. Hematol Oncol Clin North Am. 2017, 31:1045-1060. 10.1016/j.hoc.2017.08.008

2. WHO: worldwide prevalence of anaemia 1993-2005. (2008). Accessed: September 10, 2020: https://apps.who.int/iris/bitstream/10665/43894/1/9789241596657_eng.pdf.

3. WHO: global burden of iron deficiency anaemia in the year 2000 . (2002). Accessed: September 10, 2020: https://www.who.int/healthinfo/statistics/bod_irondeficiencyanaemia.pdf.

4. Carter RC, Jacobson JL, Burden MJ, et al.: Iron deficiency anemia and cognitive function in infancy . Pediatrics. 2010, 126:e427-e434. 10.1542/peds.2009-2097

5. Dobbing J: Brain, Behaviour, and Iron in the Infant Diet. Springer, London; 2013.

6. Pizarro F, Yip R, Dallman PR, Olivares M, Hertrampf E, Walter T: Iron status with different infant feeding regimens: relevance to screening and prevention of iron deficiency. J Pediatr. 1991, 118:687-692. 10.1016/S0022-3476(05)80027-7

7. Child Health Epidemiology Reference Group: Moderate to severe, but not mild, maternal anemia is associated with increased risk of small-for-gestational-age outcomes. J Nutr. 2012, 142:358-362. 10.3945/jn.111.149237

8. Haas JD, Fairchild MW: Summary and conclusions of the International Conference on Iron Deficiency and Behavioral Development, October 10-12, 1988. Am J Clin Nutr. 1989, 50:703-705. 10.1093/ajcn/50.3.703

9. Brabin BJ, Premji Z, Verhoeff F: An analysis of anemia and child mortality. J Nutr. 2001, 131:636S-648S. 10.1093/jn/131.2.636S

10. Rasmussen KM: Is there a causal relationship between iron deficiency or iron-deficiency anemia and weight at birth, length of gestation and perinatal mortality? J Nutr. 2001, 131:590-603. 10.1093/jn/131.2.590S

11. Thorsdottir I, Gunnarsson BS, Atladottir H, Michaelsen KF, Palsson G: Iron status at 12 months of age effects of body size, growth and diet in a population with high birth weight. Eur J Clin Nutr. 2003, 57:505513. 10.1038/sj.ejcn.1601594

12. Kilbride J, Baker TG, Parapia LA, Khoury SA: Incidence of iron-deficiency anaemia in infants in a prospective study in Jordan. Eur J Haematol. 2000, 64:231-236. 10.1034/j.1600-0609.2000.90037.x

13. Buonomo E, Cenko F, Altan A, Godo A, Marazzi M, Palombi L: Iron deficiency anemia and feeding practices in Albanian children. Ann Ig. 2005, 17:27-33.

14. Kumar D, Qureshi ZN, Albadwawi MS: Iron deficiency anemia in infants of Hatta suburb-UAE. Int Arch Nurs Health Care. 2019, 5:121. 10.23937/2469-5823/1510121

15. Dallman PR, Siimes MA, Stekel A: Iron deficiency in infancy and childhood. Am J Clin Nutr. 1980, 33:86118. 10.1093/ajcn/33.1.86

16. National Center for Health Statistics: National Health and Nutrition Examination Survey. (2020). Accessed: September 10, 2020: https://www.cdc.gov/nchs/nhanes/index.htm.

17. Olivares M, Walter T, Hertrampf E, Pizarro F: Anaemia and iron deficiency disease in children . Br Med Bull. 1999, 55:534-543. 10.1258/0007142991902600

18. Fishbane S, Pollack S, Feldman HI, Joffe MM: Iron indices in chronic kidney disease in the National Health and Nutritional Examination Survey 1988-2004. Clin J Am Soc Nephrol. 2009, 4:57-61. 10.2215/CJN.01670408

19. Masson C: Rheumatoid anemia. Joint Bone Spine. 2011, 78:131-137. 10.1016/j.jbspin.2010.05.017

20. Bager P, Befrits R, Wikman O, Lindgren S, Moum B, Hjortswang H, Dahlerup JF: The prevalence of anemia and iron deficiency in IBD outpatients in Scandinavia. Scand J Gastroenterol. 2011, 46:304-309. 10.3109/00365521.2010.533382

21. Stoltzfus RJ: Rethinking anaemia surveillance. Lancet. 1997, 349:1764-1766. 10.1016/S0140-6736(96)123552

22. Bentley ME, Griffiths PL: The burden of anemia among women in India . Eur J Clin Nutr. 2003, 57:52-60. 10.1038/sj.ejcn.1601504

23. Kell DB, Pretorius E: Serum ferritin is an important inflammatory disease marker, as it is mainly a leakage product from damaged cells. Metallomics. 2014, 6:748-773. 10.1039/C3MT00347G

24. Al Hawsawi ZM, Al-Rehali SA, Mahros AM, Al-Sisi AM, Al-Harbi KD, Yousef AM: High prevalence of iron deficiency anemia in infants attending a well-baby clinic in northwestern Saudi Arabia. Saudi Med J. 2015, 36:1067-1070. 10.15537/smj.2015.9.11844

25. El-Hazmi MAF, Warsy AS: The pattern for common anaemia among Saudi children. J Trop Pediatr. 1999, 45:221-225. 10.1093/tropej/45.4.221

26. Habib MA, Black K, Soofi SB, Hussain I, Bhatti Z, Bhutta ZA, Raynes-Greenow C: Prevalence and predictors of iron deficiency anemia in children under five years of age in Pakistan. A secondary analysis of National Nutrition Survey Data 2011-2012. PLoS One. 2016, 11 :e0155051. 10.1371/journal.pone.0155051

27. Population and demographic mix. (2020). Accessed: September 12, 2020: https://u.ae/en/information-andservices/social-affairs/preserving-the-emirati-national-identity/population-and-demog....

28. Wang M: Iron deficiency and other types of anemia in infants and children . Am Fam Physician. 2016, 93:270-278.

29. Baker RD, Greer FR: Diagnosis and prevention of iron deficiency and iron-deficiency anemia in infants and young children (0-3 years of age). Pediatrics. 2010, 126:1040. 10.1542/peds.2010-2576

30. Kassebaum NJ, Jasrasaria R, Naghavi M, et al.: A systematic analysis of global anemia burden from 1990 to 2010. Blood. 2014, 123:615-624. 10.1182/blood-2013-06-508325 